\title{
IRF5 promotes the proliferation of human thyroid cancer cells
}

\author{
Michele Massimino ${ }^{1 \dagger}$, Paolo Vigneri ${ }^{1 \dagger}$, Manuela Fallica ${ }^{1}$, Annamaria Fidilio ${ }^{1}$, Alessandra Aloisi ${ }^{\wedge}$, \\ Francesco Frasca ${ }^{1}$ and Livia Manzella ${ }^{1 *}$
}

\begin{abstract}
Background: Interferon Regulatory Factor 5 is a transcription factor that regulates the expression of genes involved in the response to viral infection and in the stimulation of the immune system. Moreover, multiple studies have demonstrated that it negatively regulates cell growth and oncogenesis, favoring cell differentiation and apoptosis. Thyroid carcinoma represents $98 \%$ of all thyroid malignancies and has shown a steady increase in incidence in both the USA and western European countries.

Findings: We investigated the expression, localization and function of IRF5 in thyroid cancer cells and found that it is highly expressed in both primary and immortalized thyroid carcinomas but not in normal thyrocytes. IRF5 levels were variably modulated by Interferon alpha but IRF5 only localized in the cytoplasmic compartment, thus failing to induce p21 expression as previously reported in different cell models. Furthermore, ectopic IRF5 increased both the proliferation rate and the clonogenic potential of malignant thyroid cells, protecting them from the cytotoxic effects of DNA-damaging agents. These results were directly attributable to IRF5, as demonstrated by the reduction in colony-forming ability of thyroid cancer cells after IRF5 silencing. An IRF5-dependent induction of endogenous B-Raf observed in all thyroid cancer cells might contribute to these unexpected effects.
\end{abstract}

Conclusions: These findings suggest that, in thyroid malignancies, IRF5 displays tumor-promoting rather than tumor-suppressor activities.

Keywords: IRF5, Thyroid Cancer, Cell Proliferation

\section{Background}

Interferon Regulatory Factor 5 plays essential roles in the regulation of genes induced by viral infection, cell growth, oncogenesis and apoptosis [1-8]. IRF5 was identified as a regulator of type I Interferon [9] and further studies revealed that IRF5 displays some tumor-suppressor properties as it can induce $p 21, B a k, B a x$, and Caspase 8 [10-12].

Thyroid carcinoma represents a unique model to study human carcinogenesis because it comprises tumors with different clinical and histological features [13]. Indeed, papillary and follicular thyroid cancers are slow-growing, well differentiated tumors, whereas anaplastic thyroid cancers are undifferentiated neoplasias that behave much more aggressively, usually leading to the death of the patient within one year from diagnosis $[14,15]$.

\footnotetext{
*Correspondence: manzella@unict.it

Deceased

${ }^{\dagger}$ Contributed equally

${ }^{1}$ Department of Clinical and Molecular Bio-Medicine, University of Catania, Via Androne, 83-95124 Catania, ITALY
}

We have analyzed the expression and function of IRF5 in thyroid carcinoma cells and report here multiple evidence suggesting that IRF5 may contribute to thyroid cancer proliferation and survival.

\section{Findings}

IRF5 is expressed in human thyroid cancer cells and is variably modulated by IFNa

To investigate IRF5 expression in both normal and neoplastic thyrocytes, we collected specimens derived from seven normal thyroids and three thyroid carcinomas as previously described [16]. We also examined four immortalized thyroid cancer cell lines (SW1736, WRO, 8305C and C643) all cultured as reported elsewhere [16]. An anti-IRF5 immunoblot found low or undetectable expression in normal thyrocytes. On the contrary, primary and immortalized thyroid cancer cells expressed high levels of IRF5, suggesting a possible role for this protein in thyroid carcinogenesis (Figure 1A). Interferon alpha (IFN $\alpha$ ) is a 


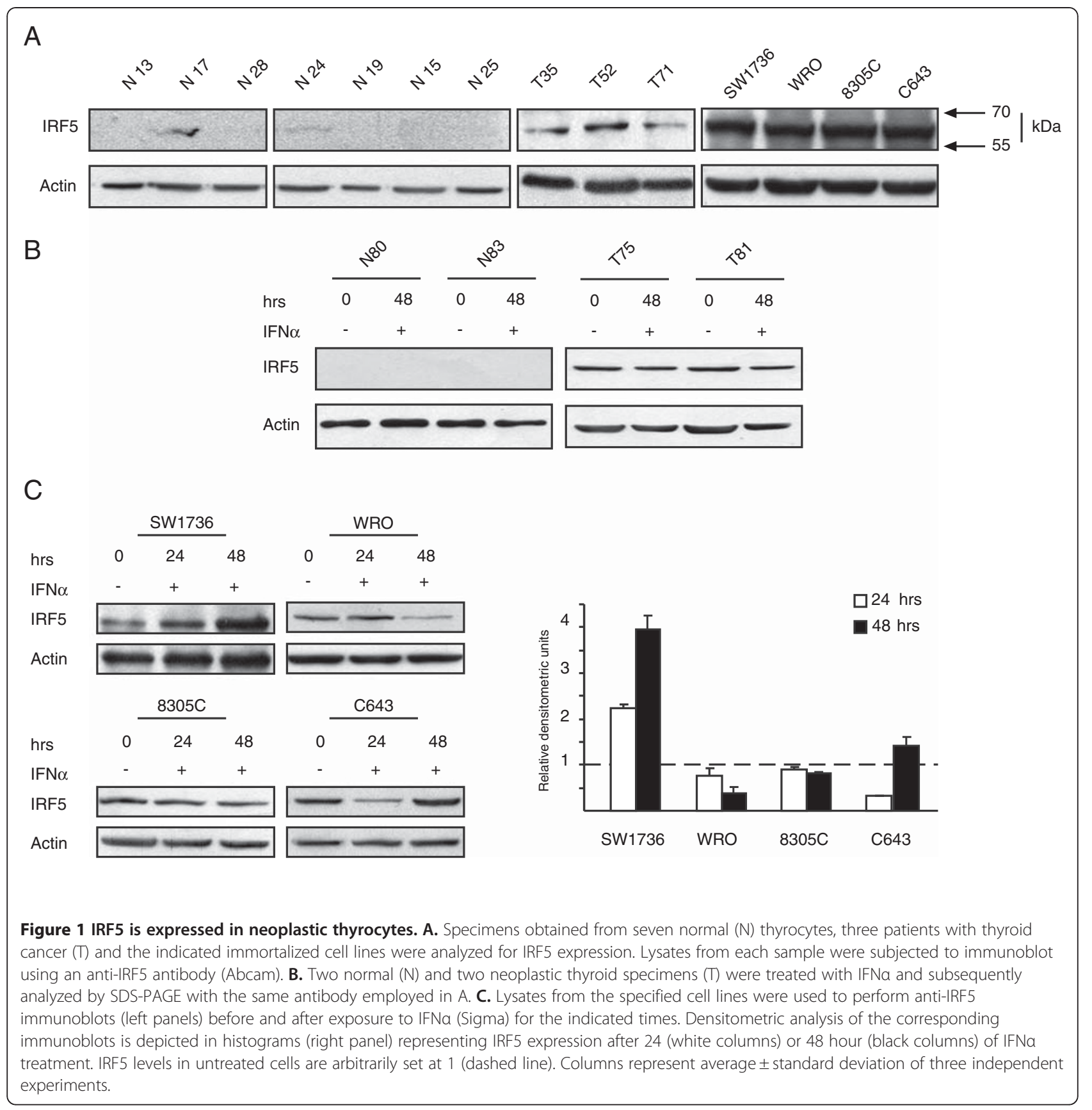

well-known transcriptional inducer of IRF5 [17]. However, its efficacy on IRF5 induction in thyroid cancer cells is still unknown. We therefore exposed normal and neoplastic thyrocytes or the above-indicated thyroid cell lines to $1000 \mathrm{U} / \mathrm{mL}$ IFN $\alpha$ and performed anti-IRF5 immunoblots. Unexpectedly, IFN $\alpha$ failed to induce IRF5 in primary thyrocytes (Figure 1B) causing instead modest variations in 8305C cells and stronger reductions - albeit with different kinetics - in WRO and C643 (Figure 1C). SW1736 were the only thyroid cancer line that displayed a robust upregulation in IRF5 expression after IFN $\alpha$.

\section{IRF5 lacks tumor-suppressor activity in thyroid} cancer cells

At steady state, IRF5 localizes to the cell cytoplasm. However, two Nuclear Localization Signals and a Nuclear Export Signal regulate IRF5 subcellular localization [18], promoting its nuclear import after viral infection [19]. To assess IRF5 intracellular distribution, we isolated nuclear and cytoplasmic fractions from four thyroid cancer cells using the Qproteom Nuclear Protein Kit (Qiagen). Immunoblots showed that IRF5 localized in the cytoplasmic compartment of each cell line, regardless of IFN $\alpha$ 


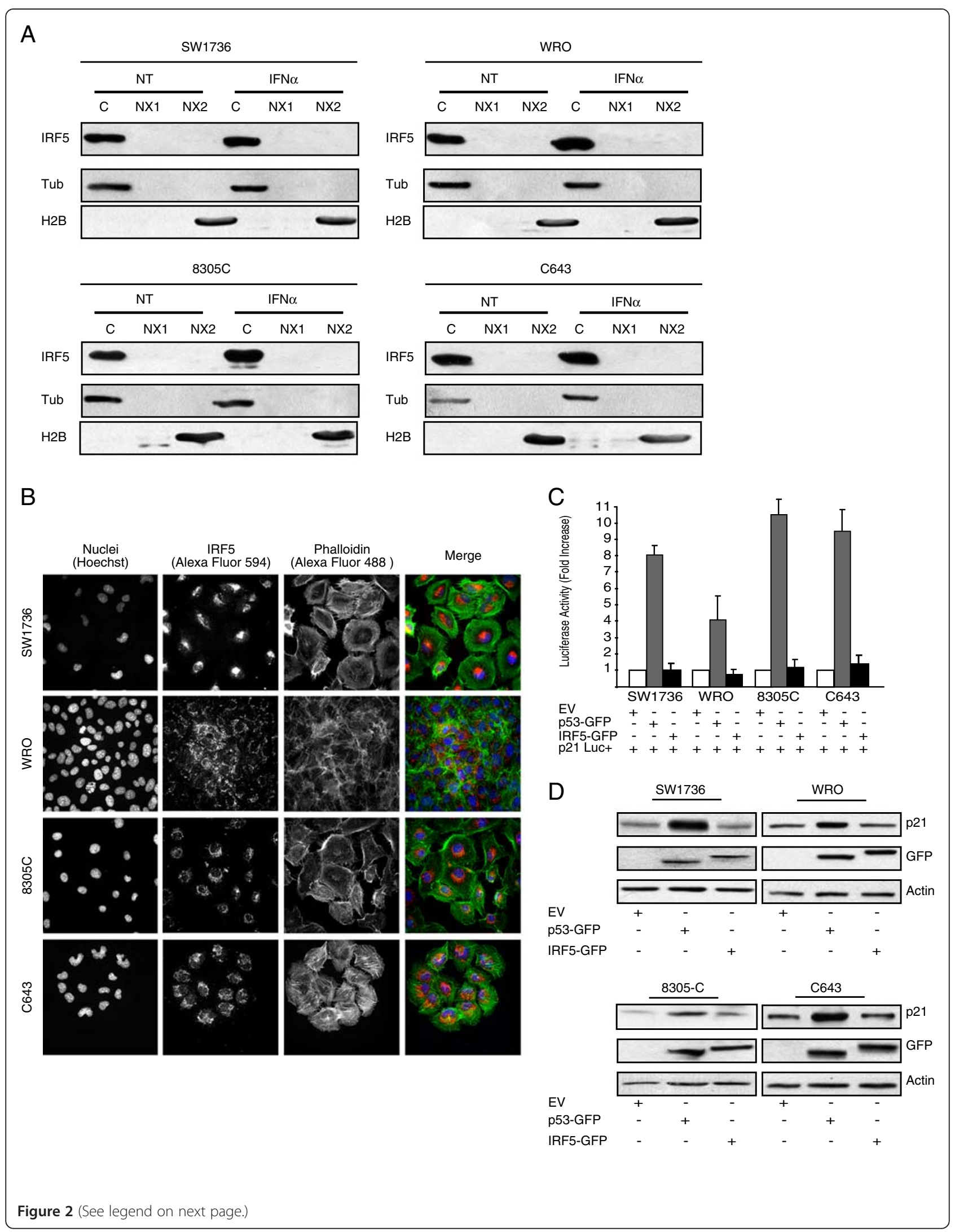


(See figure on previous page.)

Figure 2 IRF5 localizes in the cytoplasm and lacks tumor-suppressor activity in thyroid cancer cells. A. Cytoplasmic (C), soluble (NX1) and insoluble (NX2) nuclear fractions were isolated from the indicated cell lines before and after a 24 hour treatment with IFNa. The different lysates were then blotted for IRF5. Tubulin (Tub) and histone 2B (H2B) (both from Santa Cruz) confirmed the purity of cytoplasmic and nuclear extracts. B. The same cells were subjected to IF for IRF-5 using the indicated secondary antibody. Hoechst and phalloidin (Alexa Fluor 488) were employed to identify the nuclear and cytoplasmic compartments, respectively. C. Thyroid cancer cell lines were also co-transfected with a reporter construct for p21 and p53-GFP or IRF5-GFP. After 48 hours, lysates were assayed for their relative luciferase activity expressed as fold activation over control cells (p21Luc +EV, arbitrarily set at 1). Results shown represent the average of three independent experiments. D. Alternatively, the same co-transfected cells were blotted using either anti-p21 (Santa Cruz) or anti-GFP antibodies (Covance), the latter to visualize either p53 or IRF5.

treatment (Figure 2A). These findings were further confirmed by immunofluorescence (IF) experiments carried out before and after IFN $\alpha$ treatment (Figure 2B and Additional file 1).

We next wanted to establish the transcriptional activity of IRF5 on genes involved in cell-cycle progression. SW1736, WRO, 8305C, and C643 were co-transfected (using Fugene-6 from Roche) with p21-Luc (p21 promoter driving the luciferase reporter gene) and GFPIRF5 variant 3 (kindly provided by P.M. Pitha, Baltimore, $\mathrm{MD})$, p53-GFP (positive control), or an empty vector
(EV, negative control). Luciferase activity was then analyzed with the Dual-Luciferase ${ }^{\circledR}$ Reporter Assay kit (Promega). Unlike p53, IRF5 failed to induce p21-Luc activity (Figure 2C) as confirmed by anti-p21 immunoblots (Figure 2D).

\section{IRF5 induces the proliferation of thyroid cancer cells and rescues them from the effects of cytotoxic compounds}

To explore IRF5 function in thyroid cancer cells, we employed lentiviral vectors to over-express this protein in SW1736, WRO, 8305C and C643. pLEX-IRF5-GFP

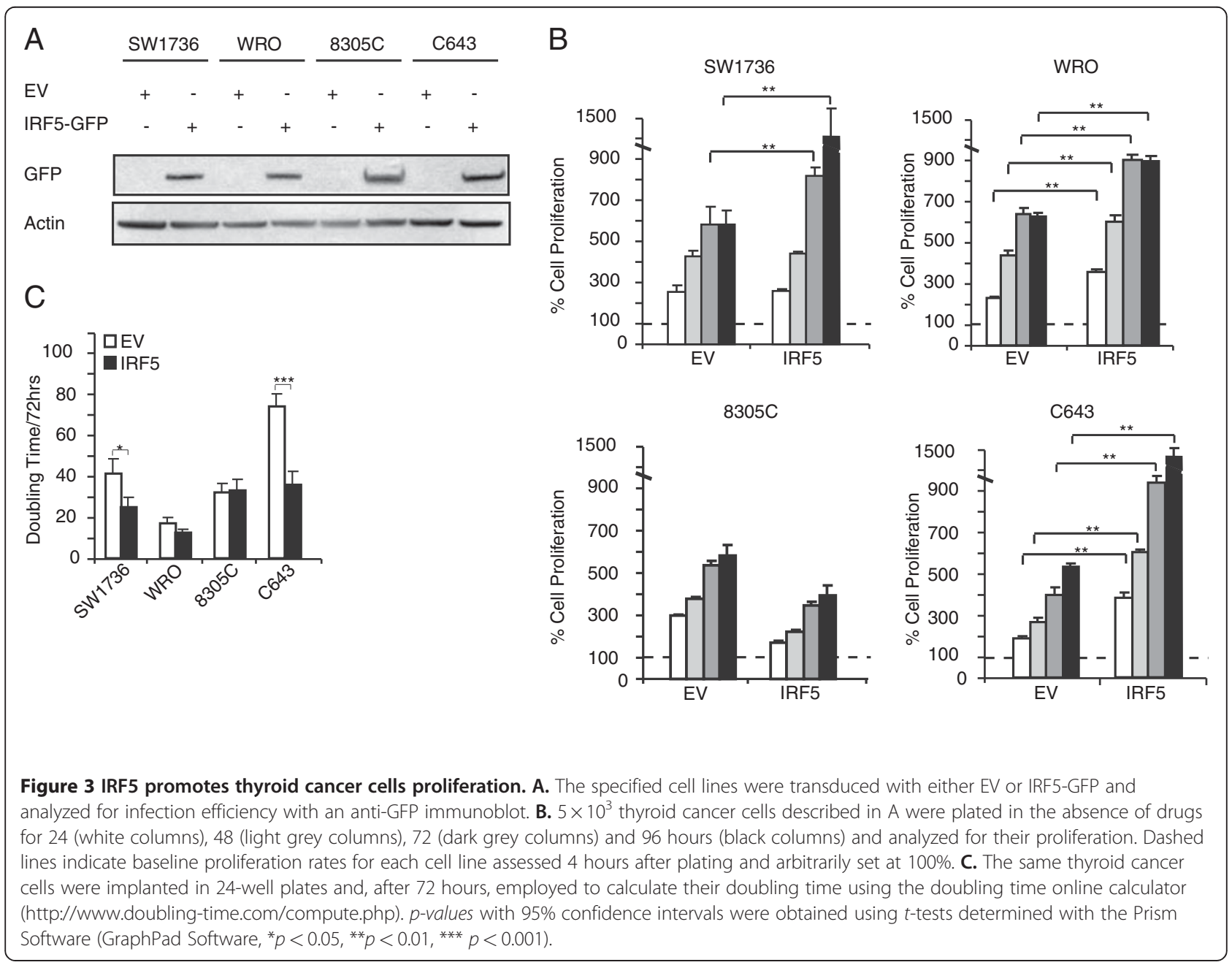


variant 3 was generated by cloning the IRF 5 cDNA fitted with SpeI-NotI adapters in the pLEX-MCS backbone (Open Biosystems, OHS4735). Transient transfection into TLA-HEK293 produced recombinant lentiviruses used to transduce thyroid cancer cells according to the Open Biosystems TLP4617 protocol. After 72 hours of puromycin selection $(2.5 \mu \mathrm{g} / \mathrm{mL})$, we performed an antiIRF5 immunoblot to confirm transgene expression (Figure 3A) and $5 \times 10^{3}$ resistant cells were placed in 96-well plates to perform ATP-Lite assays according to the manufacturer's instructions (PerkinElmer). We found that IRF5 over-expression conferred a proliferative advantage to three of four thyroid cell lines (SW1736, WRO, and C643) suggesting a role for IRF5 in promoting thyroid cancer growth (Figure 3B). These results were further confirmed by a significant increase in the proliferation rate of SW1736 and C643 in doubling time experiments with a modest effect on WRO cells (Figure 3C).

We also wanted to assess if IRF5 protected neoplastic thyroid cells from the cytotoxic effects of two DNAdamaging compounds: doxorubicin (DOXO) and cisdiamminedichloroplatinum (CDDP). $5 \times 10^{3}$ cell lines over-expressing IRF5 variant 3 were implanted in 96-well plates and left untreated or exposed to $2 \mu \mathrm{M}$ DOXO or $5 \mu \mathrm{g} / \mathrm{mL}$ CDDP. After DOXO or CDDP treatment, ATPLite assays showed increased cell numbers in SW1736,
8305 C, and C643 transduced with IRF5 as compared to the EV control. IRF5 did not affect WRO cell responsiveness to either DOXO or CDDP (Figure 4A, B).

\section{IRF5 increases both clonogenic ability and B-raf expression in thyroid cancer cells}

To establish the tumor-promoting effect of endogenous IRF5 on thyroid cancer cells, we silenced its expression using the doxycicline inducible pTRIPZ vector (shRNA anti-IRF5, gene ID NM_001098627, Open Biosystems). A non-silencing shRNA (Cat. No. RHS4743) was employed as a control. Infected thyroid cancer cells were treated with doxycicline $(1 \mu \mathrm{g} / \mathrm{mL})$ for 72 hours, lysed and blotted for IRF5 to confirm reduced expression of the transcription factor. Clonogenic assays were then performed as described above with doxycicline added every 24 hours to maintain IRF5 silencing. Reduced IRF5 expression decreased thyroid cancer colony formation implying that, in thyroid cancer cells, IRF5 facilitates single cell growth (Figure 5A).

To confirm the increased clonogenic ability of cells over-expressing IRF5, we compared clones infected with either pLEX-IRF5-GFP or pLEX-p53-GFP (inserted in the unique XhoI site of pLEX-MCS). $1 \times 10^{3}$ SW1736, WRO, C643 and $2 \times 10^{3} 8305 \mathrm{C}$ were plated in a $100 \mathrm{~mm}$ dish and fresh medium was replaced every 72 hours for 10-14 days. At this time, colonies were stained with crystal

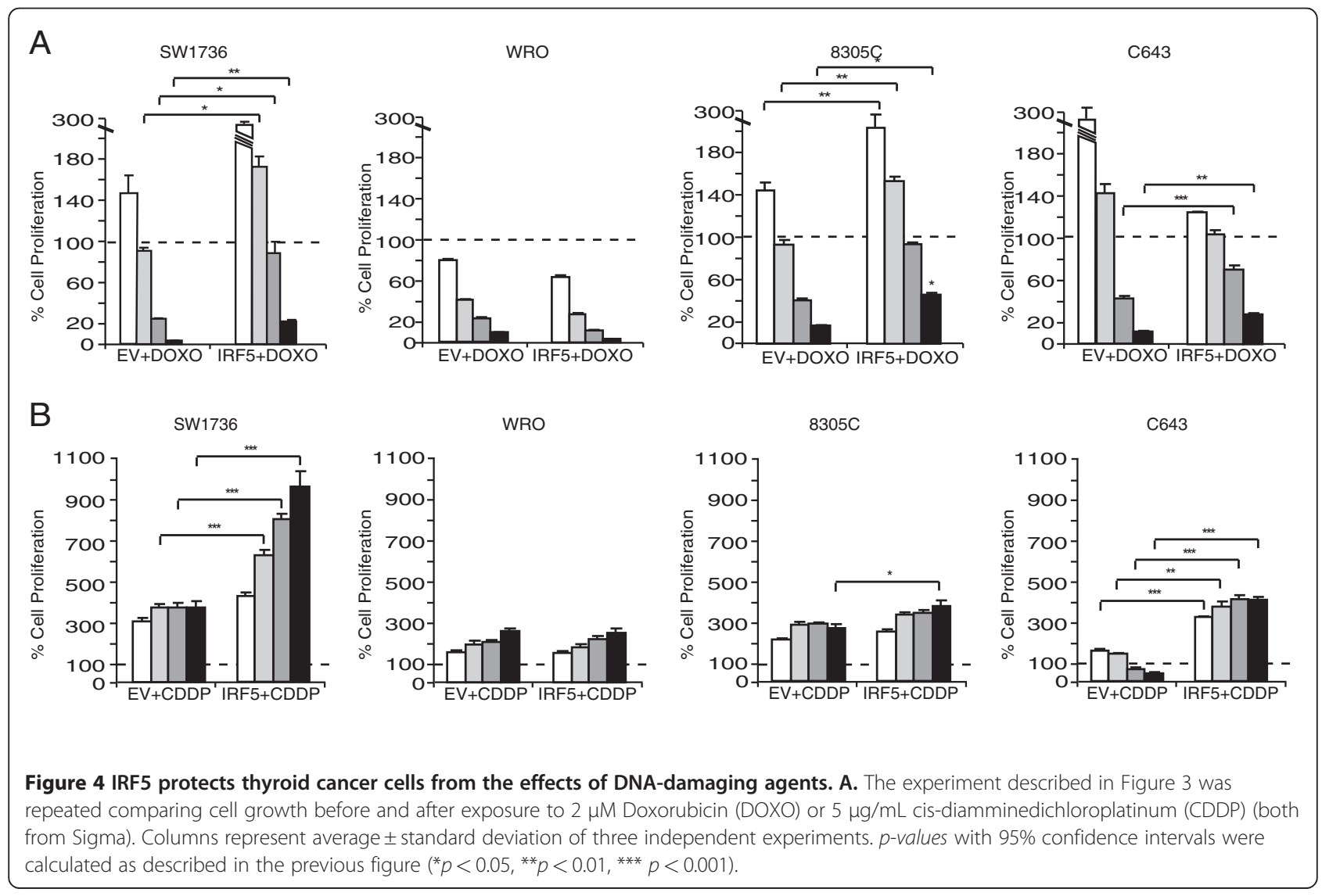



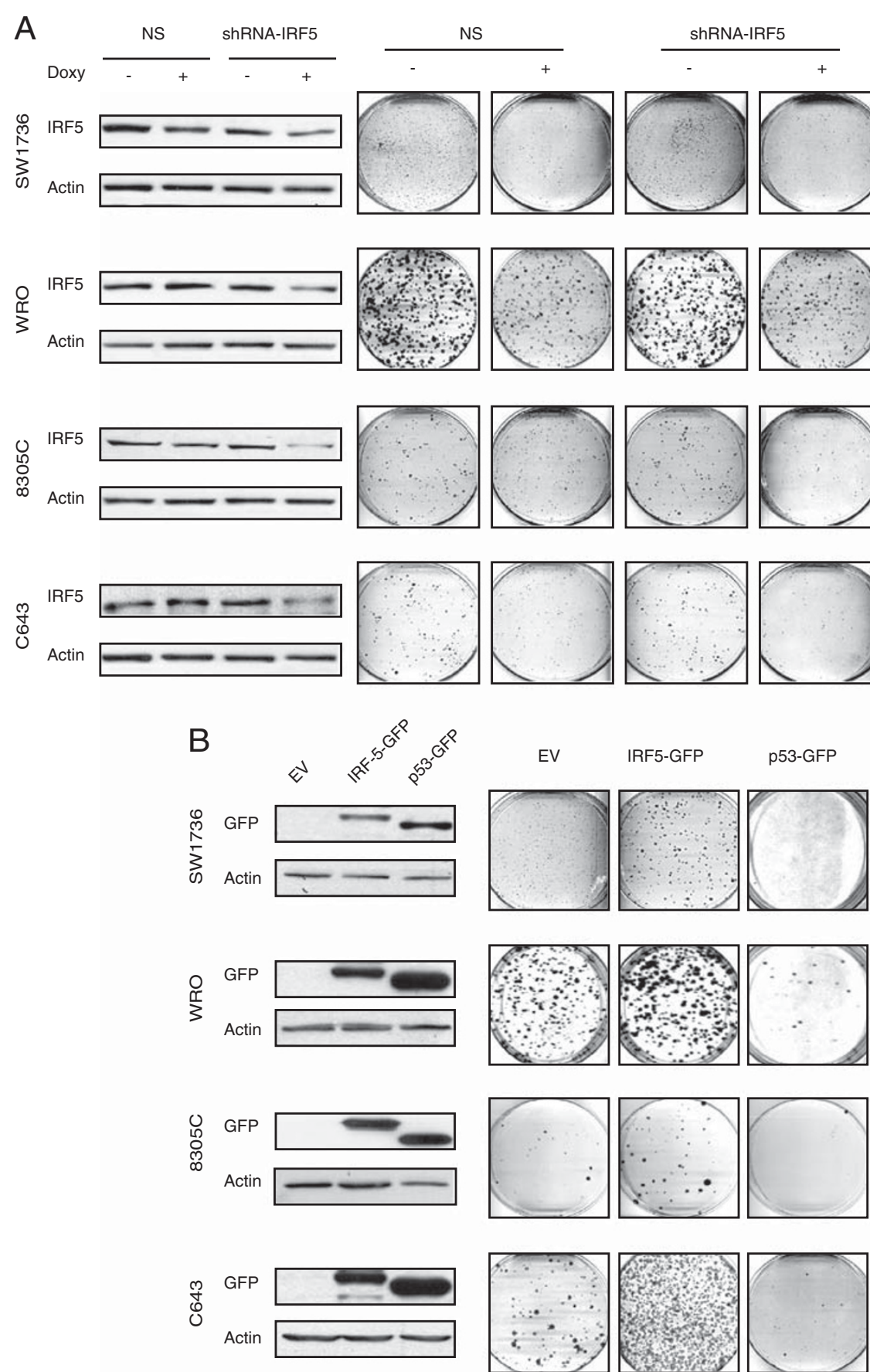

C $\quad$ SW1736 WRO $\stackrel{\text { 8305C }}{\text { C643 }}$ $\mathrm{EV}+-+-+-$ IRF5-GFP

GFP

B-Raf

Actin
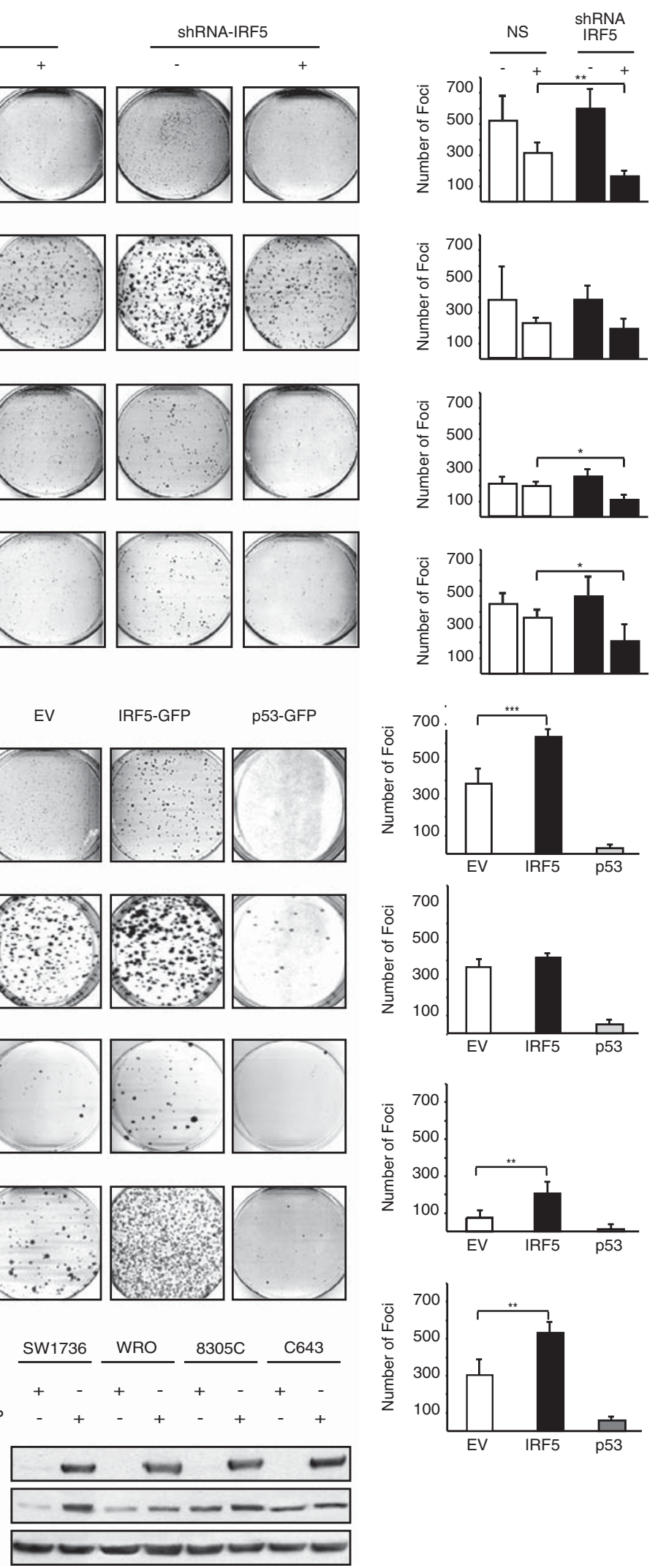

Figure 5 (See legend on next page.) 
(See figure on previous page.)

Figure 5 IRF5 over-expression increases the foci-forming capacities of thyroid cancer cells. A. Thyroid cells were infected with a doxycycline (Sigma) (Doxy)-inducible vector encoding either non-silencing or anti-IRF5 shRNA. Three days after doxycycline induction, lysates were blotted with an anti-IRF5 antibody to confirm reduction of IRF5 expression (left panels). After 1-2 weeks, infected cells were stained for their foci-forming ability (middle panels). Columns shown in the right panels represent average foci number in each plate \pm standard deviation from three separate experiments (right panels). p-values were determined as reported in Figure 3. B. Thyroid cancer cells transduced with the specified constructs were analyzed by immunoblot for the expression of IRF5-GFP or p53-GFP (left panels). After 1-2 weeks cells were stained for their foci-forming ability (middle panels). Graphs depicted in the right panels represent average foci number in each plate \pm standard deviation from three separate experiments. C. The same cells indicated in B, were analyzed for B-Raf (UPSTATE) expression by immunoblot.

violet $(0.5 \%$ in $20 \%$ Ethanol/PBS) and counted. IRF5 overexpression induced a significant increase in the total number of foci generated by three of four cell lines, suggesting that this protein improves the colony-forming ability of individual thyroid cancer cells. As expected, p53 drastically reduced colony formation compared to the $\mathrm{EV}$ control (Figure 5B).

To investigate the molecular mechanism underlying the unexpected effects of IRF5, we evaluated B-Raf expression, as this protein is mutated in $30-40 \%$ of thyroid carcinomas [20]. Interestingly, ectopic IRF5 induced endogenous B-Raf levels in all thyroid cancer cells (Figure 5C).

\section{Discussion}

In this study, we report - for the first time - that IRF5 is expressed in different thyroid carcinoma histotypes and in multiple thyroid cancer cell lines but is not detectable in normal human thyrocytes (Figure 1A). In primary normal and tumor thyroid cancer cells IFN $\alpha$ does not modulate IRF5 levels (Figure 1B) while in immortalized cell lines, IRF5 seems oddly responsive to IFN $\alpha$, since exposure to this cytokine reduces IRF5 in two thyroid cancer cells and consistently increases its expression in one cell line (Figure 1C). We found both endogenous and ectopic IRF5 in the cytoplasm of thyroid cancer cells and IFN $\alpha$ was unable to relocate the protein in the nucleus (Figure 2A, B and Additional file 2). Not surprisingly, IRF5 did not induce the p21 promoter (Figure 2C, D) [17]. On the contrary, IRF5 stimulated thyroid cancer proliferation (Figure 3B, C), protected malignant thyroid cells from the cytotoxic effects of different antiblastic compounds (Figure 4), and significantly increased their colonyforming ability (Figure 5B). Indeed, silencing of endogenous IRF5 by lentiviral shRNA reduces the clonogenic potential of most thyroid cancer cells (Figure 5A). The induction of B-Raf expression detected in thyroid cells transduced with IRF5 could partially explain its tumor-promoting effects (Figure 5C).

These findings pose the pivotal question of what role is fulfilled by IRF5 in thyroid cancer. The initial observation that the IRF5 protein is not expressed in normal thyrocytes but is detected in neoplastic thyroid cells is somewhat surprising as IRF5 has been usually associated with tumor-suppressor rather than tumor-promoting activities $[8,21,22]$. Our finding that thyroid cancer cells localize IRF5 to the cell cytoplasm implies that the protein is transcriptionally inactive. Indeed, IRF5 fails to induce a previously characterized target such as p21 and does not arrest cell-cycle progression. On the contrary, IRF5 lentiviral over-expression significantly increases the proliferation rate of malignant thyroid cells. Taken together these findings suggest two alternative scenarios: a) IRF5 might be inactivated due to point mutations or increased tyrosine phosphorylation as previously reported [22]; b) IRF5 might be either inducing the expression of tumor-promoting genes or inhibiting the promoters for tumor-suppressors. Our supplemental data seem to exclude the first scenario as direct sequencing of IRF5 revealed no point mutations and we failed to observe any variations in IRF5 tyrosine phosphorylation levels (Additional file 3). The second hypothesis seems much more likely as suggested by: i) our findings showing increased B-Raf expression in thyroid cancer cells lentivirally transduced with IRF5; ii) the protective effect of IRF5 on thyroid cells exposed to different cytotoxic drugs; iii) the reduced colony-forming potential of malignant thyrocytes displaying reduced IRF5 levels.

In summary, the present study indicates that IRF5 favors the thyroid tumoral phenotype. However, the exact mechanisms underscoring this unexpected biological function remain partially unresolved.

\section{Additional files}

Additional file 1: IRF5 shows cytoplasmic localization in thyroid cancer cells after IFNa treatment. The specified cell lines were treated with $1000 \mathrm{U} / \mathrm{mL}$ IFNa for 24 hours. Cells were then labeled for IRF5 using the indicated secondary antibody. Hoechst and phalloidin were employed to stain nuclear and cytoplasmic compartments.

Additional file 2: IRF5 v3 shows cytoplasmic localization in thyroid cancer cells. The specified cell lines were lentivirally infected with IRF5-GFP v3 and the proteins intracellular localization was analyzed by immunofluorescence.

Additional file 3: IRF5 tyrosine phosphorylation is not modulated by DNA-damaging agents in thyroid cancer cells. Whole lysates of the specified cell lines treated with $2 \mu \mathrm{M}$ Doxorubicin (DOXO) or $5 \mu \mathrm{g} /$ $\mathrm{mL}$ cisdiamminedichloroplatinum (CDDP) for the indicated times were subjected to an anti-phosphotyrosine (pY) immunoprecipitaion and 
subsequently blotted for IRF-5. G immunoglobulines (lgG) were used as a loading control.

\section{Abbreviations}

IRF5: Interferon Regulatory Factor-5; IFNa: Interferon-al pha; GFP: Green Fluorescence Protein; Luc: Luciferase; EV: Empty Vector; MCS: Multiple Cloning Site; DOXO: Doxorubicine; CDDP: Cis-diamminedichloroplatinum; shRNA: Short-Hairpin RNA; Doxy: Doxycicline.

\section{Competing interests}

The authors declare that they have no competing interests.

\section{Acknowledgements}

This work was supported by the Italian Association for Cancer Research (AIRC).

\section{Author details}

Department of Clinical and Molecular Bio-Medicine, University of Catania, Via Androne, 83-95124 Catania, ITALY.

\section{Authors' contributions}

$\mathrm{MM}, \mathrm{MF}, \mathrm{AF}$ and $\mathrm{AA}$ carried out the research. FF participated in coordination and data analysis. LM and PV designed the study and drafted the manuscript. All authors read and approved the final manuscript.

Received: 12 September 2011 Accepted: 16 April 2012

Published: 16 April 2012

\section{References}

1. Battistini A: Interferon regulatory factors in hematopoietic cell differentiation and immune regulation. J Interferon Cytokine Res 2009 29:765-780

2. Takaoka A, Tamura T, Taniguchi T: Interferon regulatory factor family of transcription factors and regulation of oncogenesis. Cancer Sci 2008 99:467-478.

3. Barnes B, Lubyova B, Pitha PM: On the role of IRF in host defense. $J$ Interferon Cytokine Res 2002, 22:59-71.

4. Manzella L, Conte E, Cocchiaro G, Guarniera E, Sciacca B, Bonaiuto C, Stagno $F$, Messina A: Role of interferon regulatory factor 1 in monocyte/ macrophage differentiation. Eur J Immunol 1999, 29:3009-3016.

5. Manzella L, Gualdi R, Perrotti D, Nicolaides NC, Girlando G, Giuffrida MA, Messina A, Calabretta B: The interferon regulatory factors 1 and 2 bind to a segment of the human c-myb first intron: possible role in the regulation of c-myb expression. Exp Cell Res 2000, 256:248-256.

6. Yanai H, Chen HM, Inuzuka T, Kondo S, Mak TW, Takaoka A, Honda K Taniguchi T: Role of IFN regulatory factor 5 transcription factor in antiviral immunity and tumor suppression. Proc Natl Acad Sci U S A 2007, 104:3402-3407.

7. Tamura T, Yanai H, Savitsky D, Taniguchi T: The IRF family transcription factors in immunity and oncogenesis. Annu Rev Immunol 2008, 26: 535-584.

8. Hu G, Barnes BJ: IRF-5 is a mediator of the death receptor-induced apoptotic signaling pathway. J Biol Chem 2009, 284:2767-2777.

9. Barnes BJ, Moore PA, Pitha PM: Virus-specific activation of a novel interferon regulatory factor, IRF-5, results in the induction of distinct interferon alpha genes. J Biol Chem 2001, 276:23382-23390.

10. Mori T, Anazawa Y, liizumi M, Fukuda S, Nakamura Y, Arakawa H: Identification of the interferon regulatory factor 5 gene (IRF-5) as a direct target for p53. Oncogene 2002, 21:2914-2918.

11. Barnes BJ, Field AE, Pitha-Rowe PM: Virus-induced heterodimer formation between IRF-5 and IRF-7 modulates assembly of the IFNA enhanceosome in vivo and transcriptional activity of IFNA genes. J Biol Chem 2003, 278:16630-16641

12. Barnes BJ, Richards J, Mancl M, Hanash S, Beretta L, Pitha PM: Global and distinct targets of IRF-5 and IRF-7 during innate response to viral infection. J Biol Chem 2004, 279:45194-45207.

13. Fagin JA: Minireview: branded from the start-distinct oncogenic initiating events may determine tumor fate in the thyroid. Mol Endocrinol 2002, 16:903-911.
14. Fagin JA: Perspective: lessons learned from molecular genetic studies of thyroid cancer-insights into pathogenesis and tumor-specific therapeutic targets. Endocrinology 2002, 143:2025-2028.

15. Giuffrida D, Gharib H: Anaplastic thyroid carcinoma: current diagnosis and treatment. Ann Oncol 2000, 11:1083-1089

16. Tirro E, Consoli ML, Massimino M, Manzella L, Frasca F, Sciacca L, Vicari L, Stassi G, Messina L, Messina A, Vigneri P: Altered expression of C-IAP1, survivin, and Smac contributes to chemotherapy resistance in thyroid cancer cells. Cancer Res 2006, 66:4263-4272.

17. Barnes BJ, Kellum MJ, Pinder KE, Frisancho JA, Pitha PM: Interferon regulatory factor 5 , a novel mediator of cell cycle arrest and cell death. Cancer Res 2003, 63:6424-6431.

18. Lin R, Yang L, Arguello M, Penafuerte C, Hiscott J: A CRM1-dependent nuclear export pathway is involved in the regulation of IRF-5 subcellular localization. J Biol Chem 2005, 280:3088-3095.

19. Barnes BJ, Kellum MJ, Field AE, Pitha PM: Multiple regulatory domains of IRF-5 control activation, cellular localization, and induction of chemokines that mediate recruitment of T lymphocytes. Mol Cell Biol 2002, 22:5721-5740

20. Salvatore G, De Falco V, Salerno P, Nappi TC, Pepe S, Troncone G, Carlomagno F, Melillo RM, Wilhelm SM, Santoro M: BRAF is a therapeutic target in aggressive thyroid carcinoma. Clin Cancer Res 2006, 12: 1623-1629.

21. Hu G, Mancl ME, Barnes BJ: Signaling through IFN regulatory factor-5 sensitizes p53-deficient tumors to DNA damage-induced apoptosis and cell death. Cancer Res 2005, 65:7403-7412.

22. Couzinet A, Tamura K, Chen HM, Nishimura K, Wang Z, Morishita Y, Takeda $\mathrm{K}$, Yagita $\mathrm{H}$, Yanai $\mathrm{H}$, Taniguchi T, Tamura T: A cell-type-specific requirement for IFN regulatory factor 5 (IRF5) in Fas-induced apoptosis. Proc Natl Acad Sci U S A 2008, 105:2556-2561.

doi:10.1186/1476-4598-11-21

Cite this article as: Massimino et al:: IRF5 promotes the proliferation of human thyroid cancer cells. Molecular Cancer 2012 11:21.

\section{Submit your next manuscript to BioMed Central and take full advantage of:}

- Convenient online submission

- Thorough peer review

- No space constraints or color figure charges

- Immediate publication on acceptance

- Inclusion in PubMed, CAS, Scopus and Google Scholar

- Research which is freely available for redistribution 